\title{
Prediction of postoperative hematoma occurrence after ultrasound-guided vacuum-assisted breast biopsy in minimally invasive surgery for percutaneous removal of benign breast lesions
}

\author{
Jianwei Zheng, Shuyan Cai, Huimin Song, Yunlei Wang, Xiaofeng Han, Gang Han, Haoliang Wu, \\ Zhigang Gao
}

Department of General Surgery, Beijing Chaoyang Hospital Affiliated with Capital Medical University, Beijing, China

Contributions: (I) Conception and design: Z Gao; (II) Administrative support: X Han; (III) Provision of study materials or patients: Y Wang; (IV) Collection and assembly of data: H Song; (V) Data analysis and interpretation: H Wu, G Han; (VI) Manuscript writing: All authors; (VII) Final approval of manuscript: All authors.

Correspondence to: Shuyan Cai. Department of General Surgery, Beijing Chaoyang Hospital Affiliated with Capital Medical University, No. 5 Jingyuan Street, Beijing 100043, China. Email: caishuyan3828@163.com.

Background: The exact factors and mechanisms involved in the development of hematoma after breast ultrasound (US)-guided vacuum-assisted biopsy (VAB) are not clear. This study aimed to analyze the occurrence, prognosis, and risk factors of hematoma after US-guided VAB for the removal of benign breast lesions.

Methods: This was a retrospective study of consecutive female patients with breast lesions (BI-RADS $3 / 4 a / 4 b$ and confirmed as benign by core needle biopsy) removed by percutaneous excisional biopsy by USguided VAB at the Beijing Chaoyang Hospital from April 2016 to December 2018. Univariable analyses were conducted to identify the factors associated with hematoma (age, nodule size, laterality, nodule location, number of nodules, BI-RADS classification of ultrasound, pathology, breast shape, menstrual period, efficacy time of bandage, and hemostatic agents). Multivariable analysis was performed to identify the factors independently associated with hematoma.

Results: A total of 293 patients (343 breast lesions) were included. Lesion removal was successful in all cases. Hematomas $\geq 1 \mathrm{~cm}$ were found in 39 patients. Finally, 38 (97.4\%) hematomas were absorbed completely within 6 months. The multivariable logistic regression analysis revealed that lesion size $(\mathrm{P}<0.001$; OR: 5.775; 95\% CI: 2.752-12.121), number of lesions ( $\mathrm{P}=0.011$; OR: 3.205, 95\% CI: 1.311-7.834), and the efficacy time of bandage 12-24 h ( $\mathrm{P}=0.003$; OR: $5.257,95 \% \mathrm{CI}$ : $1.792-15.421)$ were independently associated with hematoma occurrence after US-guided VAB excision of breast lesions. A lesion size cut-off of 23.4 mm (AUC 0.764, 95\% CI: 0.692-0.837; 59.0\% sensitivity; 80.3\% specificity; 77.5\% accuracy; $\mathrm{P}<0.001$ ) could predict hematoma occurrence.

Conclusions: US-guided excisional VAB can effectively remove benign breast lesions. The factors independently associated with postoperative hematoma are lesion size, lesion number, and effective postoperative compression time of bandage.

Keywords: Breast diseases; ultrasound; biopsy, needle; hematoma; predictive factor; minimally invasive surgery

Submitted Mar 12, 2020. Accepted for publication Aug 13, 2020.

doi: 10.21037/gs-20-344

View this article at: http://dx.doi.org/10.21037/gs-20-344 


\section{Introduction}

Up to $90 \%$ of clinical breast presentations are related to benign diseases rather than malignancies (1). USguided breast vacuum-assisted biopsy (VAB) has become a commonly used procedure for the removal of benign breast lesions (2). It is safe, well-tolerated by patients, and minimally invasive $(3,4)$, which does not require hospitalization, leaves a small scar without the need for sutures, and does not deform the breast.

Hematoma is one of the main complications after minimally invasive surgery and may limit the use of USguided VAB in some patients $(2,5,6)$. Therefore, the purpose of this study was to analyze the occurrence, prognosis, and risk factors of hematoma after US-guided $\mathrm{VAB}$ for the removal of benign breast lesions, and to provide a reference for improving the quality of this minimally invasive procedure in order to reduce hematoma formation. We present the following article in accordance with the STROBE reporting checklist (available at http:// dx.doi.org/10.21037/gs-20-344).

\section{Methods}

\section{Study design and patients}

This was a retrospective study of consecutive female patients with benign lesions removed by percutaneous excisional biopsy by US-guided VAB at the Department of General Surgery of Beijing Chaoyang Hospital from April 2016 to December 2018. The study was conducted in accordance with the Declaration of Helsinki (as revised in 2013). The study was approved by the ethics committee of Beijing Chaoyang Hospital (No. 65854). This article is a retrospective study. The need for individual consent was waived by the committee.

The inclusion criteria were: (I) high-frequency US of the breast showed single or multiple highly suspicious benign lesions; (II) breast imaging Reporting and Data System (BI-RADS) category 3, 4a, or 4b (7); (III) benign lesion confirmed by core needle biopsy; (IV) the patient complained of pain, discomfort, or anxiety from the lump, prompting lesion removal. The exclusion criteria were: (I) BI-RADS 5; (II) malignant lesion; (III) allergy to local anesthetics; or (IV) platelet aggregation inhibitors or coumarin derivatives within 7 days before the excisional biopsy.

\section{US}

The 7 G EnCor system (SenoRX, Irvine, CA, USA) was used for US-guided VAB. A Siemens Acuson Sequoia 512 US system (Siemens, Erlangen, Germany) with a $15 \mathrm{~L} 8 \mathrm{w}$ linear array probe was used. US examinations were performed by one radiologist specialized in breast imaging (15 years of experience, associate chief physician). Before the biopsy, a careful breast US examination for all lesions was performed to determine the largest diameter and direction of the lesions, then to determine the insertion site for the needle. The site was determined according to the shortest distance from the lesions, including the surrounding of the areola, the edge of the breast, or armpit. The approach to the lesion from the insertion site was along the long axis of the lesion.

\section{US-guided excisional VAB}

Freehand real-time US guidance was performed using a high-resolution linear array transducer. Initially, US guidance was performed using a color Doppler ultrasound apparatus with a $10-13 \mathrm{MHz}$ broad and linear array probe (DU-8, Esaote S.p.A., Genova, Italy). Local anesthesia was used, consisting of $1 \%$ lidocaine (used for the skin and superficial tissues, $8-12 \mathrm{~mL}$ ) and $1 \%$ lidocaine containing 1:100,000 epinephrine (used for the deeper breast tissues surround the ultrasound lesions, 15-25 mL). For masses adjacent to the pectoralis major muscle or masses just beneath the skin, lidocaine was administered between the structures and masses to artificially increase the distance for needle passage and to increase safety. In most instances of vacuum-assisted biopsies or excisions, the operations were performed by one experienced surgeon who would hold the ultrasound probe to better coordinate the whole procedure. A 3-mm skin incision was made with a \#11 blade and through which the 7-gauge EnCor device was passed and positioned just beneath the US lesion using realtime US guidance. For avoiding damage to surrounding vessels and masses located near the lesions, the probe was inserted into the bottom or the side of the lesions, and the sampling groove was pointed to the lesions. The specimens were automatically sucked into the sampling groove by negative pressure. To remove the lesions completely, the angle of the probe was adjusted, and sufficient surrounding normal breast tissue was extracted by rotating the probe. 
The size of the specimen was about $(0.3-0.5 \mathrm{~cm}) \times(1.8-$ $2.0 \mathrm{~cm}$ ) for each sample. The mean number of samples obtained from a single insertion was 10 (range, 5-18). The procedure lasted 10-30 min. The completeness of lesion excision was determined by real-time US. After blood was removed using a vacuum-assisted handheld device, manual compression to the skin incision site and probe tract was performed for approximately $10-15 \mathrm{~min}$ to ensure adequate hemostasis. After resection, a careful US examination was conducted to exclude residual tissue and obvious hematoma. Marking clips were not left at the removal site because all lesions removed were classified BI-RADS $3 / 4 a / 4 b$ or had been confirmed as benign by a previous core needle biopsy. A routine pressure dressing was placed for $12-24 \mathrm{~h}$ or 48-72 $\mathrm{h}$ with an elastic bandage, according to the surgeon's recommendations. All excisional VAB procedures were performed by the same experienced surgeons (J Zheng, S Cai). The pathological diagnosis was made according to the postoperative histological examination.

\section{Follow-up}

After 24-48 h of the procedure, all patients with benign findings on histological evaluation were required to have the first US follow-up to assess the extent of hematoma development. Thereafter, follow-up was performed at 1,3 , and 6 months. US was performed to confirm the complete removal and whether there was a recurrence. US was also performed to detect hematoma, which was defined as a hypoechoic area with an average diameter of $\geq 1 \mathrm{~cm}$ at the mastectomy site by ultrasound $24 \mathrm{~h}$ after breast VAB and accompanied by local pain, bruising, and swelling.

\section{Data collection}

Lesion size, lesion location, number of lesions, breast shape, menstrual period, efficacy time of bandage, and application of hemostatic agents during the operation were recorded from the medical charts. Basic demographics were also recorded. The patients were grouped according to the presence of a hematoma.

\section{Statistical analysis}

Data were analyzed using SPSS 20.0 (IBM, Armonk, NY, USA). Continuous data are presented as medians (range) and were analyzed using the Mann-Whitney U test. Categorical variables are presented as frequencies and percentages and were analyzed using the Pearson chisquare test or Fisher exact test, as appropriate. Two-sided P values $<0.05$ were considered to be statistically significant. Multivariable logistic regression analysis was performed on all variables with univariable $\mathrm{P}$ values $<0.05$, in order to assess the independent predictors associated with hematoma. For the multivariable analysis, binary, forward logistic regression was used. The diagnostic accuracy of the factors was assessed by receiver operating characteristic (ROC) analysis. The area under the ROC curve (AUC) with $95 \%$ confidence interval (CI) was used to assess model discrimination. It is generally accepted that an AUC of $0.7-$ 0.8 represents fair discrimination, whereas an AUC $>0.8$ represents good discrimination.

\section{Results}

\section{Characteristics of the patients and lesions}

Among 357 consecutive patients who underwent VAB, 64 were excluded because of malignant lesions; therefore, 293 patients (343 breast lesions) were included in the final analysis. There was a single breast lesion in 253 patients, two lesions in 30 patients, and three lesions in 10 patients. All patients were female. The median age was 28 years (range, 18-61 years). The median largest diameter of the 343 benign breast lesions was $18.7 \mathrm{~mm}$ (range, 9.0$35.1 \mathrm{~mm})$.

Histological evaluation showed that the lesion in the 293 patients was fibroadenoma in $43.0 \%$ (126/293), fibro adenosis in $35.2 \%$ (103/293), intraductal papilloma in $8.5 \%$ (25/293), inflammatory change in 5.8\% (17/293), retention cyst in $4.1 \%(12 / 293)$, and benign phyllodes tumor in 3.4\% (10/293). The duration of the biopsy procedure was $13-$ $45 \mathrm{~min}$ (median $20 \mathrm{~min}$ ). The number of samples removed per patient was $12-35$ (median 20). No residual lesion was detected by high-frequency US after the procedure.

\section{Hematoma}

After the first follow-up visit, 254 (88.6\%) and 39 (11.4\%) patients were without and with hematoma, respectively. Hematoma size ranged 11-42 mm (median, $19 \mathrm{~mm}$ ). Among these hematomas, the diameter of seven hematomas was $\geq 30 \mathrm{~mm}$. At the second follow-up visit, 27 hematomas were absorbed completely. The remaining seven hematomas had spontaneously disappeared at 3 months of follow-up. After 6 months of the procedure, only one residual $8 \mathrm{~mm} \times$ 
Table 1 Hematomas during follow-up after breast VAB

\begin{tabular}{lcccc}
\hline Follow-up & \multicolumn{4}{c}{ Diameter of hematoma, $\mathrm{n}(\%)$} \\
\cline { 2 - 5 } & $11-20 \mathrm{~mm}$ & $21-30 \mathrm{~mm}$ & $31-40 \mathrm{~mm}$ & $>40 \mathrm{~mm}$ \\
\hline $24 \mathrm{~h}$ & $24(61.5)$ & $8(20.5)$ & $5(12.8)$ & $2(5.2)$ \\
1 month & $5(12.8)$ & $4(10.3)$ & $2(5.1)$ & $1(2.6)$ \\
3 months & $0(0.0)$ & $2(5.2)$ & $2(5.1)$ & $1(2.6)$ \\
6 months & $0(0.0)$ & $0(0.0)$ & $0(0.0)$ & $1(2.6)$ \\
\hline
\end{tabular}

$6 \mathrm{~mm}$ hematoma was seen (Table 1).

\section{Factors for postoperative hematoma formation}

Univariable analyses showed that there were no significant associations between postoperative hematoma formation and age $(\mathrm{P}=0.463)$, right or left breast $(\mathrm{P}=0.562)$, nodule location $(\mathrm{P}=0.690)$, BI-RADS classification of ultrasound $(\mathrm{P}=0.154)$, pathology $(\mathrm{P}=0.453)$, or application of hemostatic agents $(\mathrm{P}=0.752)$ (Table 2), but hematoma was associated with lesion size $(\mathrm{P}<0.001)$, number of lesions $(\mathrm{P}=0.004)$, menstrual period $(\mathrm{P}=0.002)$, breast shape $(\mathrm{P}=0.009)$, and efficacy time of bandage $(\mathrm{P}=0.005)$ (Table 2).

Median nodule size was significantly larger in patients with hematoma occurrence compared with patients without hematoma occurrence (23.5 vs. $17.8 \mathrm{~mm}, \mathrm{P}<0.001)$. Therefore, we investigated the threshold value for nodule size that differentiated patients with postoperative hematoma formation. ROC analysis identified a cut-off point of $23.4 \mathrm{~mm}$ (AUC 0.764 , 95\% CI: 0.692-0.837; falsenegative rate of $41.0 \% ; 59.0 \%$ sensitivity; $80.3 \%$ specificity; positive predictive value of $31.5 \%$; negative predictive value of $92.7 \% ; 77.5 \%$ accuracy; $\mathrm{P}<0.001$ ) (Figure 1 ).

The multivariable logistic regression analysis showed that lesion size $(\mathrm{P}<0.001$; OR: 5.775; 95\% CI: $2.752-$ 12.121), number of lesions ( $\mathrm{P}=0.011$; OR: $3.205,95 \%$ CI: 1.311-7.834), and the efficacy time of bandage $(\mathrm{P}=0.003$; OR: $5.257,95 \%$ CI: $1.792-15.421)$ were independently associated with hematoma occurrence after VAB (Table 3).

\section{Discussion}

None of the patients in our study had any major complications. Notwithstanding, potential failures of US-guided VAB are mainly due to complications after the operation, which limits its widespread application in managing breast disease, and the major complication is hematoma occurrence. The present study showed that the occurrence of hematoma was $11.4 \%$ (39/343). This result was similar to that reported by Vargas et al. (8) but less than that reported by some previous studies $(6,9)$.

Lesion size, number of lesions, and efficacy time of bandage were independently associated with hematoma occurrence. Effective compression by the operator and application of an elastic bandage over a sufficient period of time ( $\geq 48$ hours) was a protective factor in avoiding the formation of a hematoma. VAB is a non-open surgery, and hemostasis can only be fully achieved by pressure. Pressure intensity depends on individual tolerance, hence different compression time is particularly important. This study also showed that the occurrence of hematoma decreased significantly with compression time. For lesion size, with the increase in the volume of the nodules, the residual surgical bed increases correspondingly. In addition, removing larger lesions increases the risk of vessel injury. A higher number of lesions will require an increased number of needle pass, increasing the chance of bleeding. In addition, intraoperative and postoperative considerations from the operator or the patient may lead to untimely or ineffective pressure, especially for bilateral breast surgery, which increases the chance of hematoma with the decrease in the blood viscosity and clotting. Moreover, we routinely advise patients to avoid the menstrual period when undergoing breast lesion excision. Decreased blood viscosity and coagulation function during menstruation are relative contraindications of surgery. In addition, there were two factors involved in the formation of hematoma for patients with larger and loose breasts. First, when applying a bandage to pressurize a thicker gland, the surgical area fails to get enough effective pressure effect due to the buffer effect of the gland itself. Second, the relaxation of the gland activity is larger, and enough time for effective pressure cannot be guaranteed. As supported by previous studies, the incidence rate of hematoma is markedly reduced with 
Table 2 Univariable analysis of the factors associated with postoperative hematoma occurrence $(n=293)$

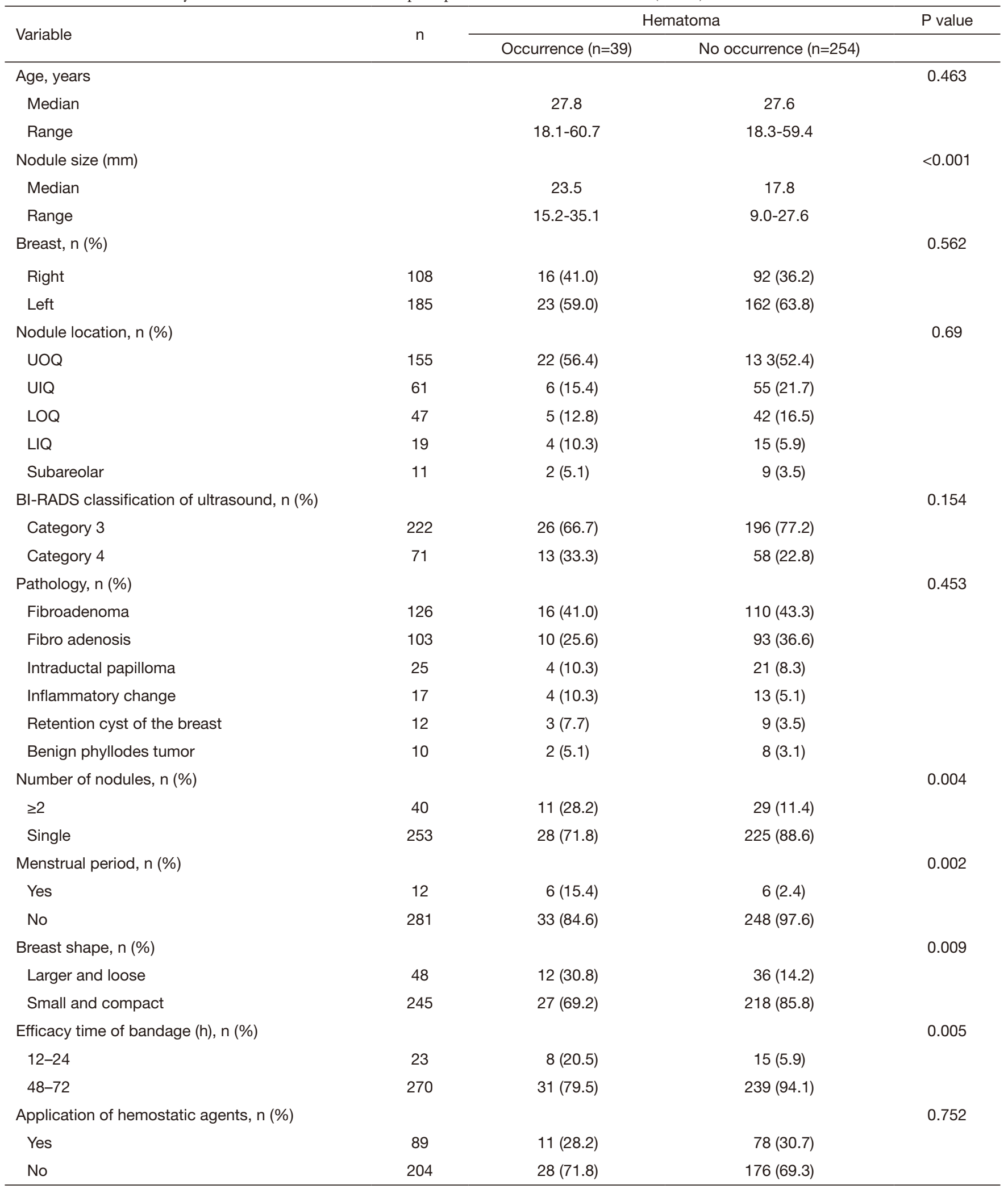

UOQ, upper outer quadrant; LOQ, lower outer quadrant; UIQ, upper inner quadrant; LIQ, lower inner quadrant; BI-RADS, breast imaging reporting and data system. 


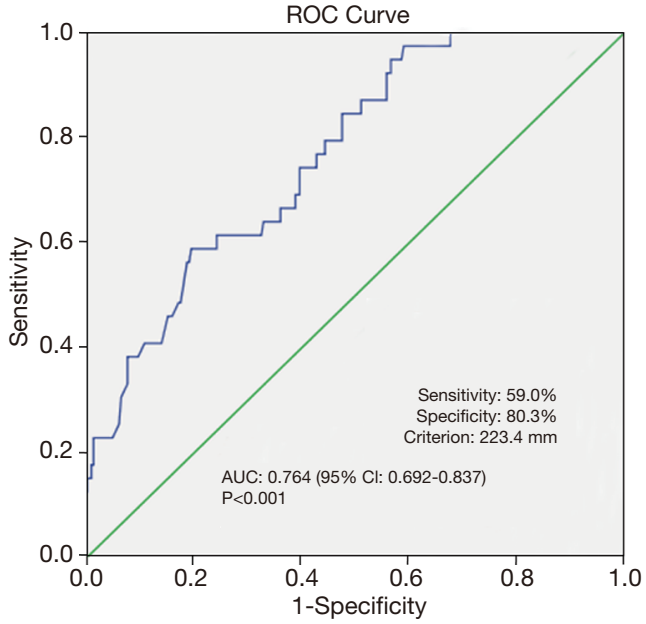

Figure 1 Receiver operating characteristics (ROC) analysis of nodule size ( $\mathrm{n}=293)$. The ROC analysis identified a cut-off point of $23.4 \mathrm{~mm}$ (area under the curve 0.764 ; sensitivity $59.0 \%$; specificity $80.3 \% ; \mathrm{P}<0.001)$.

Table 3 Multivariable analysis of influence factors associated with hematoma occurrence $(\mathrm{n}=293)$

\begin{tabular}{|c|c|c|}
\hline Variables & OR $(95 \% \mathrm{Cl})$ & $P$ \\
\hline Nodule size, mm & & $<0.001$ \\
\hline$<23.4$ & 1 & \\
\hline$\geq 23.4$ & $5.775(2.752-12.121)$ & \\
\hline Number of nodules & & 0.011 \\
\hline Single & 1 & \\
\hline$\geq 2$ & $3.205(1.311-7.834)$ & \\
\hline Efficacy time of bandage (h) & & 0.003 \\
\hline $12-24$ & 1 & \\
\hline $48-72$ & $5.257(1.792-15.421)$ & \\
\hline Menstrual period & & 0.191 \\
\hline No & 1 & \\
\hline Yes & 3.056 & \\
\hline Breast shape & & 0.341 \\
\hline Small and compact & 1 & \\
\hline Large and loose & 2.301 & \\
\hline
\end{tabular}

$\mathrm{Cl}$, confidence interval; OR, odds ratio.

the extension of bandage time $(5,10)$. Understanding the predisposing factors of hematomas is conducive to the surgeons to better grasp the indications of excisional VAB.
Regarding the postoperative hematoma, the present study showed that $69.2 \%(27 / 39)$ of the hematomas could be completely absorbed by themselves within 1 month after surgery, $87.2 \%$ (34/39) within 3 months, and $97.4 \%$ within 6 months. This suggests the safety of US-guided excisional VAB.

Our result showed that there were not only no residual tissue and recurrence, but also no obvious deformation and subsidence of breast within 6 months after the procedure. It is speculated that the rotary cutting range (i.e., the size of the lesion) will have a significant effect on the occurrence of a hematoma. Both to ensure the complete resection of the lesion while removing as little as possible of normal tissue, intraoperative US can accurately distinguish the lesion border. Besides the learning curve, the appropriate selection of the patients is a factor probably involved in hematoma occurrence. For patients with large lesions, the probability of hematoma occurrence should be told in advance to the patients. Targeted measures can be taken to prevent hematoma, such as hemostatic drug injection into the residual cavity. If bleeding is found in the wound cavity, after the hematoma is removed by vacuum aspiration, 1:20,000 adrenaline plus hemocoagulase can be injected into the wound cavity to reduce the occurrence of a hematoma. The bandage can be changed for deeper lumps. In relation to the shape of the breast, different bandage strategies can be used to compress the surgical bed as much as possible. Surgeons can more accurately predict the occurrence of hematoma in patients, which is conducive to the clinical promotion of this procedure, and reduce unnecessary open surgery for patients (11-13).

The limitation of this study is that the follow-up time was short compared with some other studies $(14,15)$. Further study in a larger patient population with benign breast lesions and long-term follow-up to assess hematoma occurrence is warranted. Nevertheless, the patients were selected, and from a single institution, and the generalizability might be limited.

From the above, US-guided excisional VAB can effectively remove benign breast lesions. The independent risk factors associated with postoperative hematoma are lesion size, number of lesions, and effective postoperative compression time of bandage.

\section{Acknowledgments}

The authors wish to thank the patients and their families for participating in the study. 
Funding: None.

\section{Footnote}

Reporting Checklist: The authors have completed the STROBE reporting checklist. Available at http://dx.doi. org/10.21037/gs-20-344

Data Sharing Statement: Available at http://dx.doi. org/10.21037/gs-20-344

Peer Review File: Available at http://dx.doi.org/10.21037/gs20-344

Conflicts of Interest: All authors have completed the ICMJE uniform disclosure form (available at http://dx. doi. org/10. 21037/gs-20-344). The authors have no conflicts of interest to declare.

Ethical Statement: The authors are accountable for all aspects of the work in ensuring that questions related to the accuracy or integrity of any part of the work are appropriately investigated and resolved. The research project has been approved by a suitably constituted Ethics Committee of the institution within which the work was undertaken and that it conforms to the provisions of in accordance with the Helsinki Declaration as revised in 2013. The study was approved by the ethics committee of Beijing Chaoyang Hospital (No. 65854). The need for individual consent was waived by the committee.

Open Access Statement: This is an Open Access article distributed in accordance with the Creative Commons Attribution-NonCommercial-NoDerivs 4.0 International License (CC BY-NC-ND 4.0), which permits the noncommercial replication and distribution of the article with the strict proviso that no changes or edits are made and the original work is properly cited (including links to both the formal publication through the relevant DOI and the license). See: https://creativecommons.org/licenses/by-nc-nd/4.0/.

\section{References}

1. Pearlman MD, Griffin JL. Benign breast disease. Obstet Gynecol 2010;116:747-58.
2. Yao F, Li J, Wan Y, et al. Sonographically guided vacuumassisted breast biopsy for complete excision of presumed benign breast lesions. J Ultrasound Med 2012;31:1951-7.

3. Debi U, Thulkar S, Sharma S, et al. Role of directional vacuum assisted breast biopsy in previously equivocal biopsies for breast masses suspicious for malignancy. Malays J Pathol 2015;37:25-33.

4. Ko KH, Jung HK, Youk JH, et al. Potential application of ultrasound-guided vacuum-assisted excision (US-VAE) for well-selected intraductal papillomas of the breast: singleinstitutional experiences. Ann Surg Oncol 2012;19:908-13.

5. Jiang $\mathrm{Y}$, Lan $\mathrm{H}, \mathrm{Ye} \mathrm{Q}$, et al. Mammotome((R)) biopsy system for the resection of breast lesions: Clinical experience in two high-volume teaching hospitals. Exp Ther Med 2013;6:759-64.

6. Luo HJ, Chen X, Tu G, et al. Therapeutic application of ultrasound-guided 8-gauge Mammotome system in presumed benign breast lesions. Breast J 2011;17:490-7.

7. Chen SC, Yang HR, Hwang TL, et al. Intraoperative ultrasonographically guided excisional biopsy or vacuumassisted core needle biopsy for nonpalpable breast lesions. Ann Surg 2003;238:738-42.

8. Vargas HI, Vargas MP, Gonzalez K, et al. Percutaneous excisional biopsy of palpable breast masses under ultrasound visualization. Breast J 2006;12:S218-22.

9. Order BM, Schaefer PJ, Peters G, et al. Evaluation of two different vacuum-assisted breast biopsy systems: Mammotome(R) system 11G/8G vs. ATEC(R) system 12G/9G. Acta Radiol 2013;54:137-43.

10. Tokumoto M, Kashiwagi S, Ishihara S, et al. Clinical experience with a handheld vacuum-assisted biopsy system (VACORA(R)) for mammary lesions. Gan To Kagaku Ryoho 2014;41:1887-9.

11. Seely JM, Verma R, Kielar A, et al. Benign Papillomas of the Breast Diagnosed on Large-Gauge Vacuum Biopsy compared with 14 Gauge Core Needle Biopsy - Do they require surgical excision? Breast J 2017;23:146-53.

12. Mosier AD, Keylock J, Smith DV. Benign papillomas diagnosed on large-gauge vacuum-assisted core needle biopsy which span $<1.5 \mathrm{~cm}$ do not need surgical excision. Breast J 2013;19:611-7.

13. Hawley JR, Lawther H, Erdal BS, et al. Outcomes of benign breast papillomas diagnosed at image-guided vacuum-assisted core needle biopsy. Clin Imaging 2015;39:576-81. 
14. Grady I, Gorsuch H, Wilburn-Bailey S. Long-term outcome of benign fibroadenomas treated by ultrasoundguided percutaneous excision. Breast J 2008;14:275-8.

15. Papathemelis T, Heim S, Lux MP, et al. Minimally Invasive

Cite this article as: Zheng J, Cai S, Song H, Wang Y, Han X, Han G, Wu H, Gao Z. Prediction of postoperative hematoma occurrence after ultrasound-guided vacuum-assisted breast biopsy in minimally invasive surgery for percutaneous removal of benign breast lesions. Gland Surg 2020;9(5):1346-1353. doi: 10.21037 /gs-20-344
Breast Fibroadenoma Excision Using an UltrasoundGuided Vacuum-Assisted Biopsy Device. Geburtshilfe Frauenheilkd 2017;77:176-81. 